\title{
Gestão hospitalar no Sistema Único de Saúde: problemáticas de estudos em política, planejamento e gestão em saúde
}

\author{
Hospital management at Brazil's National Health System: challenges \\ in the study of health policies, planning and management
}

Thadeu Borges Souza Santos (https://orcid.org/0000-0003-2497-3889) ${ }^{1}$

Andrea Laura Andrade Moreira (https://orcid.org/0000-0002-2274-3767) ${ }^{2}$

Nathália Almeida Suzart (https://orcid.org/0000-0001-7348-1341) ${ }^{1}$

Isabela Cardoso de Matos Pinto (https://orcid.org/0000-0002-1636-2909) ${ }^{2}$
${ }^{1}$ Universidade do Estado da Bahia. R. Silveira Martins 2555, Cabula. 41195001 Salvador BA Brasil. thadeu100@gmail.com

${ }^{2}$ Universidade Federal da Bahia. Salvador BA Brasil.

\begin{abstract}
The objective of this study is to present the state of the art about hospital care, from the period before the SUS until the formulation of the National Policy of Hospital Attention. This is a national and international systematic review with the adoption of Prism Protocol and survey of the scientific literature indexed in Lilacs, Scielo and Web of Science. The 157 articles included, organized in three periods, showed increase in scientific production of 2003. The findings, among other aspects, point to incipient capacity for systemic management before the creation of SUS. It is worth noting the little investment for the adjustment of the hospital care model between the 1988 Constitution and Hospital Attention Reform Plan 2003. During the period of this governmental plan and the National Policy of Hospital Attention of 2013, a set of problems led to strategies of systemic management and hospital services, such regionalization, instances of governance and adoption of indirect management models. The scientific production analyzed allowed identification of important dimensions of hospital management in SUS, from the perspective of Health Policy, Planning and Management, pointing out gaps and possibilities for the research agenda.
\end{abstract}

Key words Hospitals, Tertiary Healthcare, Policy, Planning and Management in Health
Resumo $O$ objetivo deste estudo é apresentar o estado da arte sobre gestão hospitalar, do período que antecede o SUS até a publicação da Política Nacional de Atenção Hospitalar. Trata-se de revisão sistemática nacional e internacional com adoção do Protocolo Prisma e levantamento da literatura científica indexada nas bases Lilacs, Scielo $e$ Web of Science. Os 157 artigos incluídos, organizados em três períodos históricos, evidenciaram crescimento da produção científica a partir de 2003. Os achados, dentre outros aspectos, apontam incipiente capacidade de gestão sistêmica antes da criação do SUS. Chama atenção o pouco investimento para readequação do modelo de atenção hospitalar entre a Constituição de 1988 e o Plano de Reforma da Atenção Hospitalar em 2003. No período deste plano governamental e a Política Nacional de Atenção Hospitalar de 2013, um conjunto de problemáticas conduziu estratégias de gestão sistêmica e dos serviços hospitalares, como regionalização, instâncias de governança $e$ adoção de modelos de gestão indireta. A produção científica analisada permitiu identificar importantes dimensões da gestão hospitalar no SUS, na perspectiva da Politica, Planejamento e Gestão em Saúde, apontar lacunas e lançar possibilidades para a agenda de pesquisa na área.

Palavras-chave Hospitais, Atenção Terciária à Saúde, Política, Planejamento e Administração em Saúde 


\section{Introdução}

O contemporâneo período constitucional possibilitou profundas mudanças em relação aos princípios, diretrizes, orientação política e financiamento dos sistemas e serviços de saúde. $\mathrm{Na}$ reorganização do modelo de atenção à saúde, $o$ lugar do hospital se destaca pela importância técnico-assistencial, elevado comprometimento do orçamento público alocado no setor, representação social enquanto instrumento terapêutico e redefinição enquanto ponto da rede assistencial. Como mudanças provocadas no final do século $\mathrm{XX}$, as estratégias adotadas para gestão hospitalar se fundamentaram no controle de gastos, racionalização da oferta, modernização da prestação de serviços e formulação do novo modelo de organização do cuidado à saúde nas políticas públicas ${ }^{1}$.

A área de Política, Planejamento e Gestão em Saúde (PPGS) vem contribuindo na produção de saberes e práticas, articulando investigação científica com intervenções para implementação do Sistema Único de Saúde (SUS)², exigindo confluência de conhecimentos da economia, administração, direito, ciências políticas e sociais no seu arcabouço teórico-conceitual. Uma contribuição desta área para atenção hospitalar tem sido a análise da sua complexidade quando envolve a gestão sistêmica, principalmente a partir de $1988^{3,4}$.

Importantes desafios para gestão da atenção hospitalar se destacam nestas duas primeiras décadas do Século XXI. Completando trinta anos do SUS, a literatura aponta que a complexificação sistêmica é problemática central ${ }^{5}$.

De fato, os desdobramentos identificados em estudos anteriores são muitos: a necessidade de organizar os serviços em redes assistenciais complementares e regionalizadas ${ }^{5,6}$, a exigência de maior capacidade de gestão das secretarias e conselhos de saúde ${ }^{1}$, a readequação orçamentária e uniformidade do financiamento ${ }^{5}$, sua articulação com a cadeia produtiva que o subsidia e a política de formação e alocação de recursos humanos especializados em vazios assistenciais ${ }^{1,3}$. Além desses focos, também há necessidade de qualificação dos processos de prestação de cuidados em busca da melhoria assistencial ${ }^{6}$ e superação da crônica superlotação nas salas de emergências ${ }^{7}$, a necessidade de rever a dependência de contratação complementar de serviços privados e de analisar a ampliação da adoção de estratégias de gestão publicizada $^{5,8}$.

Um balanço sobre a produção científica relacionado com as conjunturas estudadas ${ }^{4,9}$, apon- tou emergência de temáticas associadas à categoria Política de Saúde. O tema da descentralização foi muito presente entre 1974 e 2005. A partir de 1991, emergiram as problemáticas relacionadas com a Reforma Administrativa do Estado, a relação público-privada e os Modelos Alternativos de Gestão Indireta (MAGI), especificamente aqueles adotados à atenção hospitalar no SUS. E a partir de 1999, foram identificados maior frequência de estudos que tratavam sobre financiamento, modelos de gestão, regionalização de rede de serviços e economia da saúde?.

No que tange aos trabalhos incluídos na categoria planejamento e gestão, os temas em destaque foram: expansão da cobertura (a partir de 1980); propostas de reformas da gestão e organização dos serviços (a partir de 1987); mudanças das práticas político-gerenciais da esfera pública (a partir de 1991); avaliação da gestão em saúde e descentralização (a partir de 1999); e grande destaque para a temática da administração hospitalar, provavelmente pelo fomento à mudança de modelo assistencial e reorientação da sua gestão $0^{4,9}$.

Em relação à temática administração hospitalar, somente uma revisão sistemática foi identificada, abordando as práticas gerenciais ${ }^{10}$. Nela são discutidos aspectos que envolvem processo de trabalho na área hospitalar, qualidade assistencial/acreditação e importância da gestão dialógica como ferramenta inovadora no processo gerencial ${ }^{10}$. Trata-se de uma revisão sobre a gestão do serviço, especificamente.

A primeira aproximação com a busca realizada aponta a importância da atenção hospitalar para Saúde Coletiva. Na área de PPGS, faz-se importante reconhecer as evidências produzidas sobre a atenção hospitalar, para identificar lacunas que exigem novos esforços investigativos. Assim, o presente estudo tem como objetivo apresentar o estado da arte sobre gestão hospitalar, identificando problemáticas que emergem dos estudos científicos produzidos no período de busca, que envolve a década anterior à criação do SUS até à publicação da Política Nacional de Atenção Hospitalar em 2015.

\section{Método}

Realizou-se revisão sistemática nacional e internacional, cujo período de busca foi de início aberto e término em 2015, permitindo identificação de estudos anteriores da criação do SUS até a Política Nacional de Atenção Hospitalar 
(PNHOSP). Enquanto estudo qualitativo, elaborou-se protocolo de pesquisa com fontes de buscas, critérios de inclusão e exclusão, processo de tabulação e categorização dos artigos e planilha de tratamento de dados em Microsoft Excell ${ }^{\circledR}$ para estruturar apresentação dos resultados ${ }^{11}$. Durante o processo de revisão, respeitou-se o Protocolo Prisma ${ }^{12}$, cujo fluxo é apresentado na Figura 1.
Foram considerados os resumos de artigos publicados nas bases de dados da Scientific Electronic Library Online (Scielo), Literatura Latino -Americana e do Caribe em Ciências da Saúde (Lilacs) e Web of Science. Adotou-se 31 Descritores em Saúde (DECS), buscados individualmente e os critérios de inclusão foram: disponibilidade dos artigos para download, especificação do tipo de estudo e classificação dos periódicos como

Descritores em Saúde:

Administração em saúde pública; Administração de serviços de saúde; Administração hospitalar; Administração financeira de hospitais; Gestão em saúde; Parcerias público-privadas; Políticas públicas de saúde; Política, planejamento e administração em saúde; Reforma dos serviços de saúde; Sistema de saúde; Regulação e fiscalização em saúde; Atenção à saúde; Financiamento da assistência à saúde; Hospitais públicos; Hospitais sem fins lucrativos; Hospitais privados; Hospitais universitários; Hospitais federais; Hospitais estaduais; Hospitais municipais; Hospitais de ensino; Hospitais; Serviço hospitalar de emergência; Custos hospitalares; Acreditação; Sistemas locais de saúde; Sistema único de saúde; Regionalização; Saúde pública; Administração de recursos humanos em saúde; Administração de recursos humanos em hospitais.

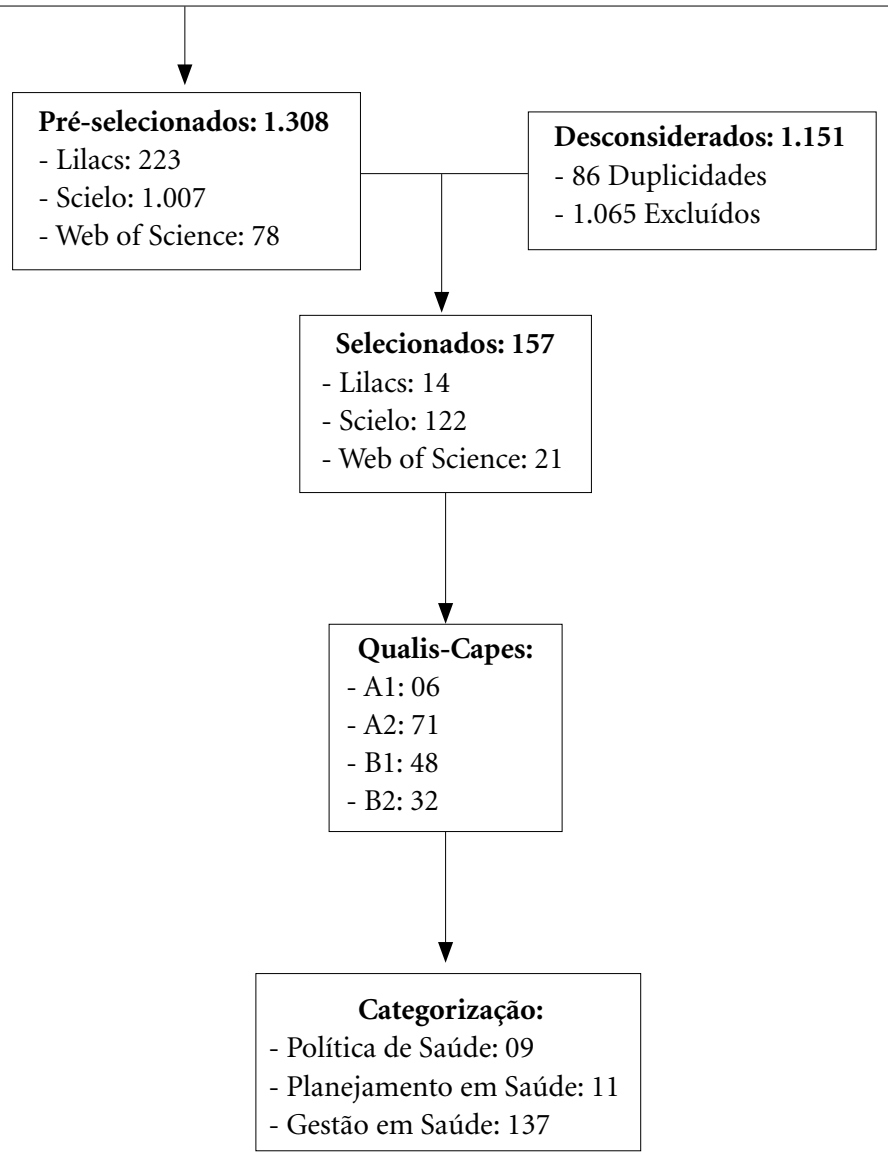

Figura 1. Fluxo do protocolo de pesquisa e tratamento da produção científica sobre gestão da atenção hospitalar no SUS 
A1, A2, B1 e B2 no Qualis-Capes 2015. Conforme respeitadas as recomendações do protocolo Prisma, o processo permite repetição do passo-a -passo e validação científica ${ }^{10}$.

O processo de tratamento dos 1308 artigos pré-selecionados exigiu caracterização conforme ano de publicação e tipo de estudo. Após leitura dos resumos, foram selecionados 157 estudos, distribuídos conforme categorias pré-estabelecidas, sendo: Política de Saúde, Planejamento em Saúde e Gestão em Saúde.

Compreende-se que política de saúde é categoria analítica que versa sobre políticas públicas nas dimensões institucional (polity), processual (politics) e material (policy) ${ }^{13}$. A concepção analítica do planejamento em saúde diz respeito aos processos de formulação, programação e operacionalização de planos, programas e ações de saú$\mathrm{de}^{14}$. Os estudos classificados na categoria gestão em saúde ${ }^{15}$, adotaram como enfoque as perspectivas dos sistemas e dos serviços, com abordagens que envolviam organização, monitoramento e avaliação em saúde. Estas concepções estruturaram o referencial teórico-analítico adotado.

Após leitura e análise, os estudos foram categorizados, classificados por ordem cronológica de publicação e distribuídos em três períodos temporais pré-estabelecidos: até criação do SUS em 1988 com a Constituição Federal do Brasil (CFB/1988); da CFB/1988 ao Plano de Reforma da Atenção Hospitalar Brasileira de 2003 (PRAHB/2003); e do PRAHB/2003 até 2015, passando pela Política Nacional de Atenção Hospitalar de 2013 (PNHOSP/2013) ${ }^{16}$.

Os documentos citados, (CFB, PRAHB e PNHOSP) expressam pontos históricos relevantes, representando respectivamente, a garantia de políticas públicas de saúde e criação do SUS, uma estratégia governamental indutora da reestruturação da atenção hospitalar com vistas às necessidades de saúde e um marco normativo de fomento em três eixos estruturantes: melhoria da gestão hospitalar, financiamento tripartite e co-responsabilização das esferas de gestão ${ }^{16}$. Esta pesquisa permitiu aproximação com a problemática e evidenciou lacunas de conhecimentos.

\section{Resultados e discussão}

O quantitativo de produção científica foi maior no Scielo (122), seguido da Web of Science (21) e Lilacs (14). Observa-se que a maioria dos artigos analisados foram publicados em periódicos classificados, segundo o Qualis-Capes 2015, como
A2 (71), seguidos por B1 (48), B2 (32), e A1 (06) (Tabela 1), apontando elevado nível de qualidade das publicações. E foi notório o crescimento da produção científica a partir de 2003, principalmente para Gestão em Saúde (Tabela 2).

Quanto aos tipos de estudos, percebe-se que a maioria se caracteriza como qualitativos, com destaque para o caráter exploratório-descritivo (88), documental (39) e de caso (16) (Tabela 3). As problemáticas evidenciadas nos artigos selecionados serão apresentadas a seguir, conforme categorias analíticas, núcleos de sentidos e respectivos períodos temporais (Quadro 1).

\section{Primeiro período: até a criação do SUS com a CFB/1988}

Conforme evidenciado na Quadro 1, os artigos até 1988 que foram incluídos na análise tiveram correlação com as categorias planejamento em saúde e gestão em saúde. Eles discutiram sobre a deficiente disponibilidade de dados e a insuficiente distribuição de leitos por especialidades hospitalares ${ }^{17}$ que aponta à baixa capacidade de planejamento da atenção hospitalar. E quanto à gestão, os problemas centrais se relacionaram com a prestação da assistência hospitalar (devido ao déficit de recursos laboratoriais e humanos e insuficiente ou má distribuição de médicos) ${ }^{18} \mathrm{e} \mathrm{a}$ baixa fidedignidade dos registros de Autorização de Internação Hospitalar ${ }^{19}$ que era o instrumento remuneratório. Consequentemente, seu inadequado preenchimento configurava frágil processo de gestão contábil da prestação de serviços públicos.

Deste primeiro período, as estratégias de formulação de uma política estruturada para atenção hospitalar foram pouco implicadas com a perspectiva de mudança do modelo de atenção à saúde. Em relação às políticas públicas ${ }^{13}$, destacou-se a inexistência de instrumentos normativos que determinassem problemas prioritários, estratégias de enfrentamento para melhoria técnico-assistencial e processos de avaliação da atenção hospitalar ${ }^{1}$. Esta falta de ferramentas de gestão sistêmica, pode indicar baixa capacidade de gestão por parte dos atores responsáveis pelo planejamento e implementação das ações voltadas para a área hospitalar.

\section{Segundo período: entre a CFB/1988 e o PRAHB/2003}

Após CFB/1988, iniciou-se o novo período democrático e o processo de formulação de po- 
Tabela 1. Distribuição quantitativa da produção científica pré-selecionada e analisada em revisão sobre gestão hospitalar no SUS, por bases Lilacs e Scielo e Qualis-Capes entre 1971 e 2015.

\begin{tabular}{|c|c|c|c|c|c|c|c|c|}
\hline \multirow{3}{*}{ Qualis } & \multicolumn{8}{|c|}{ Base de dados } \\
\hline & \multicolumn{2}{|c|}{ Lilacs } & \multicolumn{2}{|c|}{ Scielo } & \multicolumn{2}{|c|}{ Web of Science } & \multicolumn{2}{|c|}{ Total } \\
\hline & $\begin{array}{c}\text { Pré- } \\
\text { Selecionado }\end{array}$ & Analisado & $\begin{array}{c}\text { Pré- } \\
\text { Selecionado }\end{array}$ & Analisado & $\begin{array}{c}\text { Pré- } \\
\text { Selecionado }\end{array}$ & Analisado & $\begin{array}{c}\text { Pré- } \\
\text { Selecionado }\end{array}$ & Analisado \\
\hline A1 & 0 & 0 & 2 & 0 & 42 & 6 & 44 & 06 \\
\hline A2 & 25 & 2 & 216 & 60 & 30 & 9 & 271 & 71 \\
\hline B1 & 39 & 8 & 354 & 34 & 6 & 6 & 399 & 48 \\
\hline B2 & 24 & 4 & 171 & 28 & 0 & 0 & 195 & 32 \\
\hline B3 & 43 & 0 & 38 & 0 & 0 & 0 & 81 & 0 \\
\hline B4 & 4 & 0 & 10 & 0 & 0 & 0 & 14 & 0 \\
\hline B5 & 2 & 0 & 2 & 0 & 0 & 0 & 4 & 0 \\
\hline $\begin{array}{l}\text { Não } \\
\text { identificado }\end{array}$ & 86 & 0 & 214 & 0 & 0 & 0 & 300 & 0 \\
\hline Total & 223 & 14 & 1007 & 122 & 78 & 21 & 1308 & 157 \\
\hline
\end{tabular}

Fonte: Banco de dados construído pelos autores.

Tabela 2. Distribuição quantitativa da produção científica analisada em revisão sobre gestão hospitalar no SUS, por categorias analíticas e períodos históricos entre 1971 e 2015.

\begin{tabular}{|c|c|c|c|c|}
\hline \multirow[b]{2}{*}{ Categoria } & \multicolumn{4}{|c|}{ Período } \\
\hline & $<1988$ & $\begin{array}{c}1989- \\
2002\end{array}$ & $\begin{array}{c}2003- \\
2015\end{array}$ & Total \\
\hline Política de Saúde & 0 & 1 & 8 & 9 \\
\hline $\begin{array}{l}\text { Planejamento em } \\
\text { Saúde }\end{array}$ & 1 & 4 & 6 & 11 \\
\hline Gestão em Saúde & 2 & 6 & 129 & 137 \\
\hline Total & 3 & 11 & 143 & 157 \\
\hline
\end{tabular}

Fonte: Banco de dados construído pelos autores.

líticas públicas começou a ser mais propositivo com a implantação do SUS. Consequentemente, para atenção hospitalar, isto também foi percebido $^{1}$. Assim, este período entre 1989 e 2002 foi importante, pois emergiram temáticas relevantes nas três categorias analíticas.

Referente à categoria Política de Saúde, destaca-se a análise sobre os marcos normativos da reforma administrativa da saúde, especialmente das normas operacionais, que regularam a descentralização da gestão, estimularam ampliação da autonomia e da oferta/cobertura de serviços ambulatoriais e hospitalares ${ }^{20}$.

Quanto ao Planejamento em Saúde, destaca-se a profunda preocupação sobre a ampliação das atribuições dos gestores municipais a partir da descentralização da implementação de políti- cas de saúde, pois esta proposição exigiria deles maior empenho no processo de negociação ${ }^{21}$, ampliação da oferta de serviços (condicionada ao princípio da universalização) de saúde ${ }^{22}$, melhor capacidade dos governos municipais e avanço na interlocução entre os níveis estadual e municipal e reorganização da rede de serviços com base na referência e contrarreferência. Além de ser necessário superar dificuldades decorrentes da falta de recursos humanos e da inadequação da estrutura física ${ }^{23}$. Todos os aspectos referidos têm implicações para a Gestão em Saúde. Em especial, considerando a efetivação dos consórcios intermunicipais e incorporação dos líderes comunitários na gestão, com vistas à melhoria do caráter participativo e do processo de regionalização na implantação do SUS ${ }^{24}$.

Sobre os desafios da gestão sistêmica, emergiram problemáticas como crescimento da hospitalização, avanço do pré-pagamento de internações ${ }^{25}$ e emergência dos modelos de gestão indireta. Sendo que este último exigiria avanços no controle e regulação estatal ${ }^{26,27}$. E em relação a gestão dos serviços hospitalares, atenta-se para fragilidade do sistema de notificação da interna$c_{a} 0^{28} \mathrm{e}$ fomento a gestão da qualidade para efetivas mudanças na prestação de serviços ${ }^{29}$.

Com a criação do SUS, as mudanças propositivas deveriam caracterizar este período analítico com grandes implementações e readequação no modelo de atenção hospitalar. A revisão apontou que a ampliação da autonomia gestora subnacionais não foi guiada pelo modelo de atenção pensado estrategicamente e que, consequentemente, configurou uma rede desarticulada do 
Tabela 3. Distribuição quantitativa dos tipos de estudos analisados em revisão sobre gestão hospitalar no SUS, por categorias analíticas entre 1971 e 2015.

\begin{tabular}{|c|c|c|c|c|c|c|c|c|}
\hline \multirow[b]{3}{*}{ Categorias } & \multicolumn{8}{|c|}{ Tipo de estudo } \\
\hline & \multirow[b]{2}{*}{ 荀 } & \multicolumn{6}{|c|}{ Qualitativos } & \multirow[b]{2}{*}{ Total } \\
\hline & & 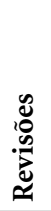 & ঠे & 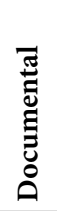 & 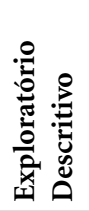 & 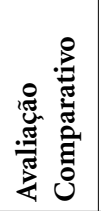 & 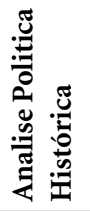 & \\
\hline Política de Saúde & 0 & 0 & 3 & 2 & 4 & 0 & 0 & 9 \\
\hline Planejamento em Saúde & 0 & 0 & 0 & 3 & 8 & 0 & 0 & 11 \\
\hline Gestão em Saúde & 2 & 1 & 13 & 34 & 76 & 9 & 2 & 137 \\
\hline Total & 2 & 1 & 16 & 39 & 88 & 9 & 2 & 157 \\
\hline
\end{tabular}

Fonte: Banco de dados construído pelos autores.

Quadro 1. Temáticas sobre política, planejamento e gestão correlacionadas com atenção hospitalar no SUS, presente na literatura científica publicada nas bases de dados Lilacs, Scielo e Web of Science entre 1971 e 2015.

\begin{tabular}{|c|c|c|c|}
\hline \multirow{3}{*}{ Categorias } & \multicolumn{3}{|c|}{ Período } \\
\hline & Primeiro & Segundo & Terceiro \\
\hline & $\begin{array}{l}\text { Até criação do SUS } \\
\text { com a CFB/1988 }\end{array}$ & $\begin{array}{c}\text { Entre } \mathrm{CFB} / 1988 \mathrm{e} \\
\text { PRAHB } / 2003\end{array}$ & $\begin{array}{c}\text { A partir da PRAHB } / 2003 \text { e } \\
\text { PNHOSP } / 2013\end{array}$ \\
\hline $\begin{array}{l}\text { 1. Política de } \\
\text { Saúde }\end{array}$ & $\begin{array}{l}\text { - Não foi } \\
\text { evidenciado }\end{array}$ & $\begin{array}{l}\text { - Descentralização e } \\
\text { autonomia dos níveis de } \\
\text { gestão através dos marcos } \\
\text { normativos da reforma } \\
\text { administrativa da saúde } \\
\end{array}$ & $\begin{array}{l}\text { - Desafios neoliberais ao SUS; } \\
\text { - Entraves do processo de } \\
\text { regionalização }\end{array}$ \\
\hline $\begin{array}{l}\text { 2. Planejamento } \\
\text { em Saúde }\end{array}$ & $\begin{array}{l}\text { - Deficiente } \\
\text { parâmetro } \\
\text { assistencial }\end{array}$ & $\begin{array}{l}\text { - Preocupações } \\
\text { relacionadas ao aumento } \\
\text { da responsabilidade de } \\
\text { implementação dos gestores } \\
\text { municipais }\end{array}$ & $\begin{array}{l}\text { - Estratégias e enfrentamentos } \\
\text { governamentais para regionalização da } \\
\text { atenção hospitalar; } \\
\text { - Complexidade da regulação em saúde }\end{array}$ \\
\hline $\begin{array}{l}\text { 3. Gestão em } \\
\text { Saúde }\end{array}$ & $\begin{array}{l}\text { - Problemas } \\
\text { relacionados } \\
\text { a assistência } \\
\text { hospitalar; } \\
\text { - Baixa } \\
\text { fidedignidade } \\
\text { de instrumentos } \\
\text { remuneratórios }\end{array}$ & $\begin{array}{l}\text { - Desafios à efetivação } \\
\text { do caráter participativo e } \\
\text { regionalização na implantação } \\
\text { do SUS; } \\
\text { - Problemáticas da gestão } \\
\text { sistêmica: crescimento } \\
\text { das hospitalizações e pré- } \\
\text { pagamento de internações } \\
\text { e emergência dos modelos } \\
\text { alternativos de gestão indireta; } \\
\text { - Problemáticas da gestão dos } \\
\text { serviços hospitalares: frágil } \\
\text { notificação de internação } \\
\text { e fomento a melhoria da } \\
\text { qualidade }\end{array}$ & $\begin{array}{l}\text { - Problemáticas do sistema hospitalar: } \\
\text { mudança para enfoque sistêmico } \\
\text { alinhado a descentralização e } \\
\text { regionalização da gestão; capacidade } \\
\text { dos gestores; regulação da rede } \\
\text { hospitalar; expansão da assistência } \\
\text { supletiva; contratação de serviços } \\
\text { complementares; ampliação da gestão } \\
\text { indireta como via de descentralização; } \\
\text { - Problemáticas da gestão dos serviços } \\
\text { hospitalares: responsabilidade entre } \\
\text { as partes nas relações público- } \\
\text { privadas, relação de poder e cultura } \\
\text { organizacional, gestão de RHS, } \\
\text { financiamento, tecnologias de } \\
\text { informação e instrumentos de gestão }\end{array}$ \\
\hline
\end{tabular}

Fonte: Banco de dados construído pelos autores. 


\section{Terceiro período: do PRAHB/2003 à PNHOSP/2013}

Os marcos históricos determinantes para a política de atenção hospitalar foram o Plano da Reforma da Atenção Hospitalar Brasileira e a PNHOSP, pois determinaram o período entre 2003 e 2013 como oportuno para a reestruturação deste nível de atenção no SUS. Intervalo de pré-decisão ${ }^{31}$ para a política pública de atenção hospitalar ${ }^{16}$.

O período iniciado em 2003, teve como principais desafios relacionados com a categoria Política de Saúde, a influência das ideias ancoradas no projeto neoliberal e a regionalização. A primeira impôs condicionantes desde o início do SUS, foi marcante na América Latina e favoreceu ampla articulação público-privado no setor saúde, configurando concepção doutrinária forte e contraria à proposta fomentada pela Reforma Sanitária Brasileira ${ }^{32,33}$. Os estudos que abordaram o tema da regionalização, destacaram a mudança do perfil epidemiológico ${ }^{34}$ e fortalecimento das instâncias loco-regionais através da superação dos problemas impostos pelo processo de municipalização da gestão $0^{35}$.

De tal modo, exigir-se-ia planejamento governamental focado nos entraves do acesso ${ }^{36}$, assim como nas estratégias de integração, regulação e financiamento da rede de serviços. E como avanços, destaca-se a governança implementada através das Comissões Intergestoras Bipartite e Regionais e Colegiados de Gestão Regional, organização das RAS e fortalecimento dos instrumentos de planejamento com o Pacto de Gestão ${ }^{37-40}$.

A categoria Planejamento em Saúde apontou duas dimensões analíticas. As estratégias governamentais para implementação da atenção hospitalar, especialmente correlacionada ao fortalecimento da regionalização. Elas consideraram os arranjos institucionais, as regras para decisão governamental, os atores inseridos na base territorial e a fundamentação legal das responsabilidades dos níveis de governo como atributos do planejamento ${ }^{41}$. E que para concretizar pro- posta de atenção hospitalar em conformidade com as RAS, dever-se-ia enfrentar os desafios da fragmentação sistêmica, complexa governança regional, problemas de acesso aos serviços de média complexidade e da necessidade de articulação política ${ }^{42-44}$. E como segunda dimensão, emergiu a problemática da regulação em saúde, destacando-se sua complexidade sócio-econômica-gerencial ${ }^{45}$ e interferência que exerce sobre a acessibilidade e equidade da rede ambulatorial e hospitalar ${ }^{46}$.

A categoria Gestão em Saúde evidenciou a gestão do sistema e também dos elementos que particularizam a gestão dos serviços hospitalares. Na primeira, os aspectos são relacionados a mudança do enfoque gestor linear ao sistêmico ${ }^{47}$, as RAS, a regionalização ${ }^{48}$, o acesso referenciado pelo tratamento ambulatorial ${ }^{49}$, a adoção da internação domiciliar ${ }^{50}$, a priorização da implantação da humanização e o acolhimento na prestação dos cuidados ${ }^{51-53}$ e dos direitos dos pacientes no SUS ${ }^{54}$.

É reconhecido que houve estímulos à regionalização e fomento à gestão colegiada na política de atenção às urgências ${ }^{55} \mathrm{e}$ a corresponsabilidade do usuário foi incorporada no aprimoramento da política de saúde ${ }^{25}$, melhorias assistenciais inclusive com adoção de protocolos assistências ${ }^{56,57}$ e mudança da representação social para mudança do modelo assistencial ${ }^{58}$.

Sobre a melhoria da capacidade dos dirigentes, destaca-se que houve incentivo aos métodos de avaliação de custo, aperfeiçoamento da capacidade operacional, adoção da lógica de economia de escala, eficiência sistêmica ${ }^{59}$, ferramentas de comparação de desempenhos e indicadores de resultados ${ }^{60} \mathrm{e}$ boas práticas gerenciais e assistenciais ${ }^{6}$.

A regulação da rede hospitalar foi destaque pela sua correspondência às internações, principalmente em UTI ${ }^{62}$, judicialização do acesso ${ }^{63} \mathrm{e}$ causas sensíveis $^{64}$. E também foi percebida como tendências: expansão da assistência supletiva, incorporação de métodos avaliativos externos à cadeia produtiva, estratégias de desospitalização e crescimento das terceirizações e das relações público-privado. De certo modo, este conjunto de fatores são condicionantes ao aumento de eficiência, garantia do acesso e via de integração entre subsetores público e privado ${ }^{65}$.

Quanto à descentralização, emergiram nos resultados a influência da concepção gerencial, da prestação de serviços complementares na rede SUS e da atribuição dos atores em situação de gestores da atenção a saúde. Assim, des- 
taca-se que a administração pública gerencial enfatiza o controle dos resultados e prestação de contas ${ }^{65}$. Relacionado às contratações de serviços com caráter complementar, evidencia-se sua sobrevivência orçamentária através do SUS e que não fortaleceram a estratégia das $\mathrm{RAS}^{66}$. O mix público-privado continua existindo devido desigualdades na oferta de serviços SUS ${ }^{67}$. E que a ampliação da responsabilidade dos gestores e prestadores foi tendência que fundamentou na garantia do direito dos usuários, melhoria da qualidade dos serviços ${ }^{68}$ e eficiência produtiva ${ }^{69}$.

A caracterização dos prestadores de serviços hospitalares se revelou como dependente de provimento do SUS, de porte majoritariamente médio e com baixa qualificação gestora e de monitoramento da qualidade assistencial ${ }^{70}$. E foi destacada a história da relação da rede filantrópica com SUS, principalmente nos municípios do interior, e sua baixa capacidade instalada de UTI, sendo que o maior porte e complexidade assistencial estão nas capitais ${ }^{71-73}$.

No caso específico da gestão indireta, atentase para o modelo de Organizações Sociais, que expandiu por limitações de recursos da década de 1990, e que enfrenta problemas como: déficit de cobertura da atenção primária, sobrecarga de atendimento com baixa complexidade e limites de referencialidade regional ${ }^{74}$. Destaca-se a importância do gestor público quanto ao controle contábil-financeiro, garantia do acesso aos serviços contratualizados ${ }^{75}$, incorporação do monitoramento, correlação custos versus necessidades sócio-demográficas, equidade e regulação ${ }^{74}$.

A gestão de serviços hospitalares, retoma crítica sobre a correlação com as naturezas pública e privada, com destaque a necessidade de determinação das responsabilidades entre os entes, enquanto partes que contratualizam e compartilham a gestão. Outras centralidades foram: relações de poder, recursos humanos, financiamento, tecnologia de informação e ferramentas de gestão ${ }^{76}$.

As relações de poder e cultura organizacional se correlacionaram. Tiveram como condicionantes: a hierarquia médica sobre as demais categorias de profissionais de saúde e administradores hospitalares ${ }^{77}$, a influência negativa da satisfação no trabalho e relações interpessoais ${ }^{78}$ e os processos de trabalhos fundamentados na burocracia profissional ${ }^{79}$, além da contemporânea reestruturação produtiva e mudança do modelo de gerenciamento $^{80,81}$.

Referente aos Recursos Humanos de Saúde (RHS), muitas considerações foram discutidas. Sobre a gestão do trabalho e educação, destaca- ram-se as influências interpessoais e infraestruturais no clima organizacional ${ }^{82,83}$. E quanto ao processo de trabalho, ampla agenda de estudos abordaram a importância da valorização profissional, utilização de indicadores de qualidade na avaliação do cuidado, definição de estratégias e metas de trabalho, avaliação de desempenho e atividade gerencial com enfoque nos custos de escalas $^{84-88}$.

Sobre o financiamento, os artigos atentaram para implantação de metodologias de análises de custos com finalidade de melhoria da transparência e busca de eficiência na gestão dos recursos públicos ${ }^{89}$. As suas problemáticas envolvem: limitação de recursos à viabilidade de execução dos contratos de gestão por OS $^{74}$ que experimentam mecanismos de flexibilização do processo de seleção e contratação; formas de remuneração e progressão funcional mais próximas das práticas adotadas pelo mercado ${ }^{90}$; e diminuição da participação orçamentária federal aos gastos com atenção especializada e hospitalar com a descentralização ${ }^{91}$.

Quanto as tecnologias de informação em saúde (TIS), merece destacar a importância dos Sistemas de Informação em Saúde (SIS), principalmente orçamentário e de internação hospitalar, enquanto ferramentas de gestão. O Sistema de Informação sobre Orçamento Público em Saúde (SIOPS) foi apresentado como relevante ao planejamento e gestão e como instrumento de controle social sobre os gastos públicos. Todavia, atenta-se para a falta de domínio na utilização da informática, desconhecimento das potencialidades que o sistema oferece e falta de regularidade na sua alimentação. Condições que levam a não utilização amplamente pelos gestores ${ }^{92}$. Enquanto que o Sistema de Informações Hospitalares (SIH) permite correlacionar as internações hospitalares, o quantitativo de serviços prestados, cobertura territorial e demanda de usuários ${ }^{93}$. Deve-se atentar que a assistência hospitalar é um elemento motriz do processo de incorporação tecnológica do complexo industrial da saúde ${ }^{94}$.

Sobre os instrumentos de gestão especificamente, aparecem processos de tomada de decisão utilizando o SIH, através de colegiados gestores e núcleos de epidemiologia de hospitais $^{95-99}$; a adoção de avaliação de desempenho na busca por melhor prestação de contas públicas ${ }^{100,101}$, eficiência e eficácia ${ }^{102}$; racionalização e dispensação de insumos conforme organização da cadeia produtiva $^{103}$; instrumentalização dos processos terapêuticos, reorganização infraestrutural, tecnologias de acolhimento e fortalecimento dos 
fluxos internos e regulatórios frente às superlotações das emergências ${ }^{104-106}$; rastreamento de eventos adversos ${ }^{107}$; e adoção de engenharia clínica no controle dos custos envolvendo equipamentos e tecnologias ${ }^{108}$.

Outro instrumento de gestão importante é a acreditação por ser ferramenta de avaliação externa da qualidade gerencial e assistencial que favorece processos de mudança na cultura organizacional ${ }^{109-111}$.

\section{Considerações Finais}

A estratégia governamental de reorganização da atenção hospitalar no SUS avançou, destacadamente após 2003 com o plano de sua reforma. A análise crítica da Saúde Coletiva não foi distante ou ingênua neste processo, sendo evidenciada vasta problemática neste estudo, à luz da Política, Planejamento e Gestão em Saúde.

Destacam-se no estudo as vias de descentralização administrativa, regionalização da atenção e capacidade de gestão sistêmica e dos serviços. Os artigos analisados apontaram diferentes dimensões que refletem a complexidade e os desafios da atenção hospitalar no SUS, seja pelos frágeis instrumentos de gestão e regulação estatais, hegemônico modelo de atenção hospitalocêntrico ou pela necessidade de avanços na implementação da concepção sistêmica e participativa. Tais elementos, junto às suas particularidades relacionadas ao financiamento, recursos humanos e corresponsabilidades contratuais da relação público-privada, permitiram responder à ques- tão de pesquisa com duas importantes considerações.

A primeira, corresponde aos resultados da categoria política de saúde. Compreendeu-se que, mediante concorrente desafio entre as correntes neoliberal e societal, a descentralização avançou no sentido da responsabilização da gestão hospitalar ao nível estadual e, administrativamente, ao novo setor público não-estatal. Esta condição aponta uma nova institucionalidade jurídica da atenção hospitalar no SUS e mostra o quanto é centrada no gestor estadual, pactuada com os níveis federal e municipal/regional e contratualizada com modelos alternativos de gestão indireta.

A segunda consideração é relacionada à categoria analítica Planejamento em Saúde, que sinalizou aumento da responsabilidade dos gestores e que, em consequência da relação público-privada no SUS, esta complexifica ainda mais o planejamento e gestão sistêmica.

Assim, uma agenda de pesquisas sobre atenção hospitalar no campo da Saúde Coletiva pode ser pensada. $O$ modelo de gestão contratualizada seria realmente favorável à expansão da cobertura assistencial estatal com readequação do modelo de atenção a saúde? Quais contribuições (de fato) os modelos alternativos de gestão indireta trazem à gestão da rede e serviços hospitalares? Qual a capacidade regulatória estatal, no processo de implementação da atenção hospitalar, para a gestão contratualizada? Estas são novas questões que emergem e devem conformar uma agenda de pesquisa, uteis para guiar futuros esforços investigativos na área de Política, Planejamento e Gestão da atenção hospitalar no SUS.

\section{Colaboradores}

TBS Santos trabalhou na concepção, metodologia, pesquisa, tratamento de dados, análise e redação final. NA Suzart trabalhou na pesquisa, tratamento e análise. ALA Moreira trabalhou na pesquisa, tratamento e análise. ICM Pinto trabalhou na concepção, metodologia, análise e redação final. 


\section{Referências}

1. Braga Neto FC, Barbosa PR, Santos IS, Oliveira CMF. Atenção hospitalar: evolução histórica e tendências. In: Giovanella L, Escorel S, Lobato LVC, Noronha JC, Carvalho AI, organizadores. Politicas e sistema de saúde no Brasil. Rio de Janeiro: Fiocruz; 2012. p. 577608.

2. Schraiber LB, Peduzzi M, Sala A, Nemes MIB, Castanheira ERL, Kon R. Planejamento, gestão e avaliação em saúde: identificando problemas. Cien Saude Colet 1999; 4(2):221-242.

3. Teixeira CF, Jesus WLA, Souza MKB, Rocha MND. Produção cientifica sobre política, planejamento e gestão em saúde no campo da saúde coletiva: visão panorâmica. In: Paim JS, Almeida Filho N, organizadores. Saúde coletiva: teoria e prática. Rio de Janeiro: MedBook; 2014. p.585-594.

4. Souza MKB, Teixeira CF. Produção científica sobre gestão de sistemas de saúde: um estudo realizado em espaço Web (1987-2009). Cien Saude Colet 2012; 17(4):935-944.

5. Vecina Neto G, Malik AM. Tendências na assistência hospitalar. Cien Saude Colet 2007; 12(4):825-839.

6. Portela MC. Accountability e qualidade. Cien Saude Colet 2007; 12(3):840-848.

7. Solla JJSP, Chioro A. Atenção ambulatorial especializada. In: Giovanella L, Escorel S, Lobato LVC, Noronha JC, Carvalho AI, organizadores. Políticas e sistema de saúde no Brasil. Rio de Janeiro: Fiocruz; 2012. p. 547-576.

8. Coelho I. Os hospitais no Brasil. São Paulo: HUCITEC; 2016.

9. Paim JS, Teixeira CF. Política, planejamento e gestão em saúde: balanço do estado da arte. Rev Saude Publica 2006; 40(n. esp.):73-78.

10. Farias DC, Araújo FO. Gestão hospitalar no Brasil: revisão da literatura visando ao aprimoramento das práticas administrativas em hospitais. Cien Saude Colet 2017; 22(6):1895-1904.

11. Minayo MCS. Análise qualitativa: teoria, passos e fidedignidade. Cien Saude Colet 2012; 17(3):621-626.

12. Urrútia G, Bonfill X. Declaración PRISMA: uma propuesta para mejorar la publicación de revisiones sistemáticas y metanalisis. Med Clin (Barc) 2010; 135(11):507-511.

13. Paim JS. Políticas de saúde no Brasil. In: Rouquayrol MZ, Almeida Filho N. Epidemiologia e Saúde. 6a ed. Rio de Janeiro: MEDSI; 2003. p. 587-603.

14. Teixeira CF, Jesus WLA, Souza MKB, Rocha MND. Produção científica sobre política, planejamento e gestão em saúde no campo da saúde coletiva: visão panorâmica. In: Paim JS, Almeida N, organizadores. Saúde Coletiva. Teoria e prática. Rio de Janeiro: MedBook; 2014. p.585-594.

15. Teixeira C, Silveira P. Glossário de análise política em saúde. Salvador: EdUFBA; 2017.

16. Santos TBS, Pinto ICM. Política Nacional de Atenção Hospitalar: con(di)vergências entre normas, Conferências e estratégias do Executivo Federal. Saude Debate 2017; 41(n. esp. 3):99-113.

17. Pugliese C, Loureiro S, Santos ACS, Padilha HP, Souza JM, Lessa I, Serra G, Codes J, Dias CN, Araújo JD. Inquérito sobre assistência hospitalar e morbidade hospitalar no município do Salvador (Bahia), Brasil. Rev Saude Publica 1971; 5(1):1-16.
18. Yunes J. Situação da assistência médico-sanitária e hospitalar no estado do Ceará, Brasil. Rev Saude Publica 1973; 7(2):123-138.

19. Lebrão ML. Análise da fidedignidade dos dados estatísticos hospitalares disponíveis na Secretaria de Estado da Saúde de São Paulo em 1974. Rev Saude Publica 1978; 12(2):234-249.

20. Viana ALD, Heimann LS, Lima LD, Oliveira RG, Rodrigues SH. Mudanças significativas no processo de descentralização do sistema de saúde no Brasil. Cad Saude Publica 2002; 18(Supl.):139-151.

21. Tanaka OY, Escobar EMA, Giménez ASM, Camargo KG, Lelli CLS, Yoshida TM. Gerenciamento do setor saúde na década de 80, no Estado de São Paulo, Brasil. Rev Saude Publica 1992; 26(3):185-194.

22. Schneider A. Os consórcios intermunicipais de saúde no estado do Rio de Janeiro. Physis: Rev Saúde Colet 2001; 11(2):51-66.

23. Monnerat GL, Senna MCM, Souza RG. A reorganização dos serviços de saúde no cenário local. Cien Saude Colet 2002; 7(3):509-521.

24. Vázques ML. Participação social nos serviços de saúde: concepções dos usuários e líderes comunitários em dois municípios do Nordeste do Brasil. Cad. Saude Publica 2003; 19(2):579-591.

25. Rocha JSY, Simões BJG. Estudo da assistência hospitalar pública e privada em bases populacionais, 19861996. Rev Saude Publica 1999; 33(1):44-54.

26. Machado CV. Novos modelos de gerencia nos hospitais públicos: as experiências recentes. Physis 2001; 1(1):105-197.

27. Ibanhes LC, Heimann LS, Junqueira V, Boaretto RC, Pessoto UC, Cortizo CT, Castro IEN, Rocha JL, Kayano J, Luiz OC, Barboza R, Telesi Junior E. Governança e regulação na saúde: desafios para a gestão na Região Metropolitana de São Paulo, Brasil. Cad Saude Publica 2007; 23(3):575-584.

28. Deslandes SF, Silva CMFP. Análise da morbidade hospitalar por acidentes de trânsito em hospitais públicos do Rio de Janeiro, RJ, Brasil. Rev Saude Publica 2000; 34(4):367-372.

29. Fausto MCR. Limites e impasses atuais da municipalização da saúde: a experiência de Cabo Frio. Physis 2001; 11(2):67-94

30. Campos GWS, Amaral MA. A clínica ampliada e compartilhada, a gestão democrática e redes de atenção como referenciais teórico-operacionais para a reforma do hospital. Cien Saude Colet 2007; 12(4):849-859.

31. Kingdon JW. Agendas, alternatives and public policies. $2^{\mathrm{a}}$ ed. United States of America: Addson-Wesley Longman; 1995.

32. Campos CMS, Viana N, Soares CBS. Mudanças no capitalismo contemporâneo e seu impacto sobre as políticas estatais: o SUS em debate. Saúde Soc 2015; 24(Supl. 1):82-91.

33. Sestelo JAF, Souza LEPF, Bahia L. Saúde suplementar no Brasil: abordagens sobre a articulação público/ privada na assistência à saúde. Cad Saude Publica 2013; 29(5):851-866.

34. Contel FB. Os conceitos de região e regionalização: aspectos de sua evolução e possíveis usos para a regionalização da saúde. Saúde Soc 2015; 24(2):447-460. 
35. Spedo SM, Tanaka OU, Pinto NRS. O desafio da descentralização do Sistema Único de Saúde em município de grande porte: o caso de São Paulo, Brasil. Cad Saude Publica 2009; 25(8):1781-1790.

36. Lima SML, Rivera FJU. A contratualização nos Hospitais de Ensino no Sistema Único de Saúde brasileiro. Cien Saude Colet 2012; 17(9):2507-2521.

37. Dourado DA, Elias PEM. Regionalização e dinâmica política do federalismo sanitário brasileiro. Rev Saúde Publica 2011; 45(1):204-211.

38. Kuschnir R, Chorny AH. Redes de atenção à saúde: contextualizando o debate. Cien Saude Colet 2010; 15(5):2307-2316.

39. Silva EC, Gomes MHA. Regionalização da saúde na região do Grande $\mathrm{ABC}$ : os interesses em disputa. Saúde Soc 2014; 23(4):1383-1396.

40. Santos AM, Giovanella L. Regional governance: strategies and disputes in health region management. Rev Saude Publica 2014; 48(4):622-631.

41. Menicucci TMG. O Sistema Único de Saúde, 20 anos: balanço e perspectivas. Cad Saude Publica 2009; 25(7):1620-1625.

42. Shimizu HE. Percepção dos gestores do Sistema Único de Saúde acerca dos desafios da formação das Redes de Atenção à Saúde no Brasil. Physis 2013; 23(4):11011122.

43. Spedo SM, Pinto NRS, Tanaka OY. O difícil acesso a serviços de média complexidade do SUS: o caso da cidade de São Paulo, Brasil. Physis 2010; 20(3):953-972.

44. Lima JC, Rivera FJU. Gestão de sistemas regionais de saúde: um estudo de caso no Rio Grande do Sul, Brasil. Cad Saude Publica 2006; 2(10):2179-2189.

45. Gamarra TPN. Contribuições epistemológicas da ergologia para a regulação em saúde. Trab Educ Saúde 2014; 12(3):483-498.

46. Ferreira JBB, Mishima SM, Santos JS, Forster AC, Ferraz CA. O complexo regulador da assistência à saúde na perspectiva de seus sujeitos operadores. Interface (Botucatu) 2010; 14(33):345-358.

47. Borba GS, Kliemann Neto FJ. Gestão Hospitalar: identificação das práticas de aprendizagem existentes em hospitais. Saúde Soc 2008; 17(1):44-60.

48. Barreto Junior IF. Regionalização do atendimento hospitalar público na Região Metropolitana da Grande Vitória, ES. Saúde Soc 2015; 24(2):461-471.

49. Perrechi MCT, Ribeiro AS. Tratamento de tuberculose: integração entre assistência hospitalar e rede básica na cidade de São Paulo. J Bras Pneumol 2009; 35(11):1100-1106.

50. Silva KLS, Sena R, Leite JCA, Seixas CT, Gonçalves AM. Internação domiciliar no Sistema Único de Saúde. Rev Saude Publica 2005; 39(3):391-397.

51. Carneiro MF, Iriart JAB, Menezes GMS. "Largada sozinha, mas tudo bem": paradoxos da experiência de mulheres na hospitalização por abortamento provocado em Salvador, Bahia, Brasil. Interface (Botucatu) 2013; 17(45):405-418.

52. Aquino EMI, Menezes G, Barreto-de-Araújo TV, Alves MT, Alves SV, Almeida MCC, Schiavo E, Lima LP, Menezes CAS, Marinho LFB, Coimbra LC, Campbell O. Qualidade da atenção ao aborto no Sistema Único de Saúde do Nordeste brasileiro: o que dizem as mulheres? Cien Saude Colet 2012; 17(7):1765-1776.
53. Gusmão-Filho FAR, Carvalho EF, Araújo Junior JLAC. Avaliação do grau de implantação do Programa de Qualificação da Atenção Hospitalar de Urgência (Qualisus). Cien Saude Colet 2010; 15(Supl. 1):12271238.

54. Gomes AMA, Sampaio JJC, Carvalho MGB, Nations MK, Alves MSCF. Código dos direitos e deveres da pessoa hospitalizada no SUS: o cotidiano hospitalar na roda de conversa. Interface (Botucatu) 2008; 12 (27):773-782.

55. O'Dwyer G. A gestão da atenção às urgências e o protagonismo federal Cien Saude Colet 2010; 15(5):23952404.

56. O’Dwyer G, Matta IEA, Pepe VLE. Avaliação dos serviços hospitalares de emergência do estado do Rio de Janeiro. Cien Saude Colet 2008; 13(5):1637-1648.

57. Soares VMN, Souza KV, Azevedo EMM, Possebon CR, Marques FF. Causas de mortalidade materna segundo níveis de complexidade hospitalar. Rev Bras Ginecol Obstet 2012; 34(12):536-543.

58. Oliveira LH, Mattos RA, Souza AIS. Cidadãos peregrinos: os "usuários" do SUS e os significados de sua demanda a prontos-socorros e hospitais no contexto de um processo de reorientação do modelo assistencial. Cien Saude Colet 2009; 14(5):1929-1938.

59. Souza PC, Scatena JHG. É economicamente viável regionalizar a atuação de um hospital público de médio porte? Physis 2010; 20(2):571-590.

60. Lins ME, Lobo MSC, Silva ACM, Fiszman R, Ribeiro VJP. O uso da Análise Envoltória de Dados (DEA) para avaliação de hospitais universitários brasileiros. Cien Saude Colet 2007; 12(4):985-998.

61. Lima SML, Rivera FJU. A contratualização nos Hospitais de Ensino no Sistema Único de Saúde brasileiro. Cien Saude Colet 2012; 17(9):2507-2521.

62. Evangelista PA, Barreto SM, Guerra HL. Central de regulação de leitos do SUS em Belo Horizonte, Minas Gerais, Brasil: avaliação de seu papel pelo estudo das internações por doenças isquêmicas do coração. Cad Saude Publica 2008; 24(4):767-776.

63. Vargas MAO, Ramos FRS, Schneider DG, Schneider N, Santos AC, Leal SMC. Internação por ordem judicial: dilemas éticos vivenciados por enfermeiros. Rev Gaúcha Enferm. 2013; 34(1):119-125.

64. Fernandes VBL, Caldeira AP, Faria AA, Rodrigues Neto JF. Internações sensíveis na atenção primária como indicador de avaliação da Estratégia Saúde da Família. Rev Saude Publica 2009; 43(6):928-936.

65. Ermel RC, Bigio FM, Evangelista AFR, Gomes MFP, Fracolli LA. Algumas perspectivas para análise da gestão da saúde no estado de São Paulo (Brasil). Cien Saude Colet 2011; 16(3):1899-1906.

66. Alburquerque MSV, Morais HMM, Lima LP. Contratualização em saúde: arena de disputa entre in teresses públicos e privados. Cien Saude Colet 2015; 20(6):1825-1834.

67. Santos IS, Ugá MAD, Porto SM. O mix público-privado no Sistema de Saúde Brasileiro: financiamento, oferta e utilização de serviços de saúde. Cien Saude Colet 2008; 13(5):1431-1440.

68. Matos CA, Pompeu JC. Onde estão os contratos? Análise da relação entre os prestadores privados de serviços de saúde e o SUS. Cien Saude Colet 2003; 8(2):629-643. 
69. Cesconetto A, Lapa JS, Calmo MCM. Avaliação da eficiência produtiva de hospitais do SUS de Santa Catarina, Brasil. Cad Saude Publica 2008; 24(10):24072417.

70. Portela MC, Lima SML, Ugá MAD, Gerschman S, Vasconcellos MTL. Estrutura e qualidade assistencial dos prestadores de serviços hospitalares à saúde suplementar no Brasil. Cad. Saúde Pública 2010; 26(2):399408.

71. Portela MC, Lima SML, Barbosa PR, Vasconcellos MM, Ugá MAD, Gerschman S. Caracterização assistencial de hospitais filantrópicos no Brasil. Rev Saude Publica 2004; 38(6):811-818.

72. Lima SML, Barbosa PR, Portela MC, Ugá MAD, Vasconcellos MM, Gerschman S. Caracterização gerencial dos hospitais filantrópicos no Brasil. Cad Saude Publica 2004; 20(5):1249-1261.

73. Cunha JAC, Correa HL. Avaliação de desempenho organizacional: um estudo aplicado em hospitais filantrópicos. RAE 2013; 53(5):485-499.

74. Tiberio AA, Souza EM, Sarti FM. Considerações sobre Avaliação de Estabelecimentos de Saúde sob Gestão de OSS: o caso do Hospital Geral do Grajaú. Saúde Soc 2010; 19(3):557-568.

75. Carneiro Junior N, Elias PE. Controle público e equidade no acesso a hospitais sob gestão pública não estatal. Rev Saude Publica 2006; 40(5):914-920.

76. Lorenzetti J, Lanzoni GMM, Assuiti LFC, Pires DEP, Ramos FRS. Gestão em saúde no Brasil: diálogo com gestores públicos e privados. Texto Contexto Enferm 2014; 23(2):417-425.

77. Ferreira LCM, Garcia FC, Vieira A. Relações de poder e decisão: conflitos entre médicos e administradores Hospitalares. Rev Adm Mackenzie 2010; 11(6):31-54.

78. Carvalho MC, Rocha FLR, Marziale MHP, Gabriel CS, Bernardes A. Valores e práticas de trabalho que caracterizam a cultura organizacional de um hospital público. Texto Contexto Enferm 2013; 22(3):746-753.

79. Vaghetti HH, Padilha MICS, Lunardi Filho WD, Lunardi VL, Costa CFS. Significados das hierarquias no trabalho em hospitais públicos brasileiros a partir de estudos empíricos. Acta Paul Enferm 2011; 24(1):8793.

80. Bernardes A, Cummings G, Évora YDM, Gabriel CS. Contextualização das dificuldades resultantes da implementação do Modelo de Gestão Participativa em um hospital público. Rev Latino-Am Enferm 2012; 20(6):1-10.

81. Brito MJM, Melo MCOL, Monteiro PRR, Costa JO. Interfaces das mudanças hospitalares na ótica da enfermeira-gerente. Rev Adm Empres 2004; 44(n. esp.):34-47.

82. Nishide VM, Benatti MCC, Alexandre NMC. Ocorrência de acidente do trabalho em uma unidade de terapia intensiva. Rev Latino-Am Enferm 2004; 12(2):204-211.

83. Vargas MAO, Ramos FRS, Schneider DG, Schneider N, Santos AC, Leal SMC. Internação por ordem judicial: dilemas éticos vivenciados por enfermeiros. Rev Gaucha Enferm 2013; 34(1):119-125.

84. Danet A, March JC, Romera IG. Comunicação, participação e liderança na percepção do clima emocional em um hospital universitário da Andaluzia, Espanha. Cad Saude Publica 2014; 30(3):546-558.
85. Kalisch BJ, Labelle AE, Boqin X. Trabalho em equipe e tempo de resposta às chamadas de enfermagem: estudo exploratório. Rev Latino-Am Enferm 2013; 21(n. esp.):1-9.

86. Silveira TVL, Prado Júnior PP, Siman AG, Amaro MOF. Opinião dos enfermeiros sobre a utilização dos indicadores de qualidade na assistência de enfermagem. Rev Gaúcha Enferm 2015; 36(2):82-88.

87. Hausmann M, Peduzzi M. Articulação entre as dimensões gerencial e assistencial do processo de trabalho do enfermeiro. Texto Contexto Enferm 2009; 18(2):258-265.

88. Balbueno EA, Nozawa MR. Levantamento dos tipos de repercussões resultantes da avaliação de desempenho em enfermagem hospitalar. Rev Latino-Am Enferma 2004; 12(1):58-64.

89. Bonacim CAG, Araújo AMP. Gestão de custos aplicada a hospitais universitários públicos: a experiência do Hospital das Clínicas da Faculdade de Medicina de Ribeirão Preto da USP. RAP 2010; 44(4):903-931.

90. Barbosa NB. Regulação do trabalho no contexto das novas relações público versus privado na saúde. Cien Saude Colet 2010; 15(5):2497-2506.

91. Machado CV, Lima LD, Viana ALD, Oliveira RG, Iozzi FL, Albuquerque MV, Scatena JHG, Mello GA, Pereira AMM, Coelho APS. Federalismo e política de saúde: comissões intergovernamentais no Brasil. Rev Saude Publica 2014; 48(4):642-650.

92. Silva KSB, Bezerra AFB, Sousa IMC, Gonçalves RF. Conhecimento e uso do Sistema de Informações sobre Orçamentos Públicos em Saúde (SIOPS) pelos gestores municipais, Pernambuco, Brasil. Cad Saude Publica 2010; 26(2):373-382.

93. Tomimatsu MFAI, Andrade II SM, Soares DA, Mathias TAF, Sapata MPM, Soares DFPP, Souza RKT. Qualidade da informação sobre causas externas no Sistema de Informações Hospitalares. Rev Saude Publica 2009; 43(3):413-420.

94. Barbosa PR, Gadelha CAG. O papel dos hospitais na dinâmica de inovação em saúde. Rev Saude Publica 2012; 46(Supl.):68-75.

95. Mendes MFM, Freese E, Guimarães MJB. Núcleos de epidemiologia em hospitais de alta complexidade da rede pública de saúde situados no Recife, Pernambuco: avaliação da implantação. Rev Bras Saúde Matern Infant 2004; 4(4):435-442.

96. Pitilin EB, Gutubir D, Molena-Fernandes CA, Pelloso SM. Internações sensíveis à atenção primária específicas de mulheres. Cien Saude Colet 2015; 20(2):441448.

97. Pinheiro Filho FP, Sarti FM. Falhas de mercado e redes em políticas públicas: desafios e possibilidades ao Sistema Único de Saúde. Cien Saude Colet 2012; 17(11):2981-2990.

98. Bernardes A, Cecilio LCA, Évora YDM, Gabriel CS, Carvalho MB. Modelo de gestão colegiada e descentralizada em hospital público: a ótica da equipe de enfermagem. Rev Latino-Am Enferm 2011; 19(4):1-8.

99. Cassiani SHB, Teixeira TCA, Opitz SP, Linhares JC. O sistema de medicação nos hospitais e sua avaliação por um grupo de profissionais. Rev Esc Enferm USP 2005; 39(3):280-287. 
100. Bonacim CAG, Araújo AMP. Influência do capital intelectual na avaliação de desempenho aplicada ao setor hospitalar. Cien Saude Colet 2010; 15(Supl. 1):1249-1261.

101. Gonçalves AC, Noronha CP, Lins MPE, Almeida RMVR. Análise Envoltória de Dados na avaliação de hospitais públicos nas capitais brasileiras. Rev Saude Publica 2007; 41(3):427-435.

102. Tanaka OU, Tamaki EM. O papel da avaliação para a tomada de decisão na gestão de serviços de saúde. Cien Saude Colet 2012; 17(4):821-828.

103. Infante $M$, Santos MAB. A organização do abastecimento do hospital público a partir da cadeia produtiva: uma abordagem logística para a área de saúde. Cien Saude Colet 2007; 12(4):945-954.

104. Bittencourt RJ, Hortale VA. Intervenções para solucionar a superlotação nos serviços de emergência hospitalar: uma revisão sistemática. Cad Saude Publica 2009; 25(7):1439-1454.

105. Gonçalo CR, Borges ML. Organizações de Saúde Intensivas em Conhecimento: um estudo no contexto de serviços de alta complexidade. Saúde Soc 2010; 19(2):449-461.

106. Guedes MVC, Henriques ACPT, Lima MMN. Acolhimento em um serviço de emergência: percepção dos usuários. Rev Bras Enferm 2013; 66(1):31-37.

107. Mendes W, Travassos C, Martins M, Marques PM. Adaptação dos instrumentos de avaliação de eventos adversos para uso em hospitais brasileiros. Rev Bras Epidemiol 2008; 11(1):55-66.

108. Souza DB, Milagre ST, Soares AB. Avaliação econômica da implantação de um serviço de Engenharia Clínica em hospital público brasileiro. Rev Bras. Eng Bioméd 2012; 28(4):327-336.

109. Fortes MTR, Baptista TWF. Acreditação: ferramenta ou política para organização dos sistemas de saúde? Acta Paul Enferm 2012; 25(4):626-631.

110. Feldman LB, Cunha ICKO. Identificação dos critérios de avaliação de resultados do serviço de enfermagem nos programas de acreditação hospitalar. Rev Latino-Am Enferm 2006; 14(4):540-545.

111. Schiesari LMC. Avaliação externa de organizações hospitalares no Brasil: podemos fazer diferentes? Cien Saude Colet 2014; 19(10):4229-4234.

Artigo apresentado em 16/08/2017

Aprovado em 20/12/2018

Versão final apresentada em 22/12/2018 
